\title{
Beiträge zur partiellen Hydrolyse der Cellulose. \\ ron
}

\section{Dr. Géza Zemplén.}

(Aus dem chemischen Institut der Hochschule fär Forstwesen is Selmeczbanja.) (Der Redaktion zugegangsa am 17. April 1913.)

Seitdem Skraup und König ${ }^{1}$ ) bei der partiellen Hydrolyse der Cellulose in den Besitz der schön krystallisierenden Cellobiose gelangten, haben sich mehrere Forscher') mit den Bildungsbedingungen und mit den Eigenschaften dieses interessanten Disaccharids beschäftigt. Dabei wurde die partielle Hydrolyse der Cellulose immer durch gleichzeitige Einwirkung von konzentrierter Schwefelsäure und Essigsäureanhydrid ausgeführt.

Ich habe mir die Aufgabe gestellt, zu untersuchen, wie sich die Cellulose verhält, wenn man sie der getrennten Einwirkung der beiden Agentien aussetzt. Demnach wollte ich die durch Schwefelsäure allein gebildeten Abbauprodukte der Cellulose mit Essigsäureanhydrid acetylieren. Bei der letzteren Operation war mir die Anwendung der konzentrierten Schwefelsäure wieder unvermeidlich, und deshalb hat sich die Frage wie folgt modifiziert:

Wie verhält sich die mit Schwefelsäure zuvor partiell hydrolysierte Cellulose unter den Bedingungen der Acetolyse, die aus normalen Cellulosen zur Bildung von Oktacetylcellobiose führen?

Bei der Einwirkung der starken Schwefelsäure werden gewisse Bindungen im Cellulosemolekül langsam gelöst und es entsteht eine Reihe von Produkten, deren erstes, faßbares Glied Amyloid genannt wird. Diese Substanz bildet sich auf der Ober-

1) Zd. Skraup und J. Köriig, Monatshefte für Chemie, Bd. 22. S. 1011 (1901).

2) Maquenne und Goodvin, Bulletin de la société chimique [3], Bd. 31, S. 854 (1907). - Zd. Skraup, Über Stärke, Glykogen und Cellulose. - E. Geins perger, Monatshefte für Chemie, Bd. 26, S. 1415 (1905). Emil Fischer und Géza Zemplén, Liebigs Annalen, Bd. 365, S. 1 (1909); Bd. 372, S. 254 (1910). - Ber. d. Deutsch. chem. Ges., Bd. 43, S. 2536 (1910). - W. Schliemann, Liebigs Annalen, Bd. 378, S. 366 bis 381 (1911). - Friedrich Klein, Zeitschrift f. angewandte Chemie, Bd. 25, S. 1409 (1.012). 
fläche der Pergamentpapiere und wird von einigen Forschern mit der Hydrocellulose identisch gehalten. ${ }^{1}$ ) Die Hydrocellulose gibt bei der Acetolyse ebensoviel Oktacetylcellobiose, wie die typische Cellulose, und so ist man imstande, durch Anwendung der Acetolyse zu entscheiden, ob die Identifikation des Amyloid mit der Cellulose begründet ist oder nicht. Meine Versuche zeigen, daß das von mir untersuchte Amyloid tatsächlich bei der Acetolyse Oktacetylcellobiose liefert, ihre Menge ist aber viel geringer als diejenige, die aus Hydrocellulosen gewöhnlich entsteht. Demnach ist das Amyloid mit der Hydrocellulose nicht identisch. Es ist wahrscheinlich ein Gemisch aus verschiedenen depolymerisierten Produkten, unter denen vielleicht auch Hydrocellulose vorkommt. Das Resultat stimmt mit den Untersuchungen von C. Schwalbe ${ }^{2}$ ) überein, der einen Unterschied zwischen Amyloid und Hydrocellulose auf Grund des Reduktionsvermögens feststellte. Allerdings scheint das nach dem Verfahren von Flechsig dargestellte Amyloid tiefer abgebaut zu sein, als die Hydrocellulose.

Zur Untersuchung gelangten zwei weitere Produkte, die ich bei einer längeren Einwirkung ( 2 bezw. 6 Stunden nach völliger Lösung der Cellulose) von Schwefelsäure auf Cellulose erhielt. Ich war bereit, bei der Acetolyse dieser tiefer abgebauten Präparate ${ }^{3}$ ) entweder kleine krystallisierte Acetylderivate, oder vielleicht $\alpha$-Pentacetylglukose zu erhalten. Die Versuche verliefen aber ganz anders. In beiden Fällen gewann ich Oktacetylcellobiose, und die Menge derselben war nahezu gleich derselben, die aus Amyloid gebildet war.

Die Untersuchung zeigt d.emnach, daß durch die Einwirkung der Schwefelsäure aus Cellulose Pro-

1) Diese Ansicht wird durch Girard vertreten. Weiteres über die Abbauprodukte der Cellulose durch Schwefelsäure siehe Garl Schwalbe, Die Chemie der Zellulose, 1911-1912.

2) C. Schwalbe, Zeitschr. f. angewandte Chemie, Bd. 20, S. 2166 bis 2172 (1907).

3) Max König und Stanislau Schubert, Über Ätherschwefelsäuren einiger Kohlenhydrate, Monatshefte für Chemie, Bd. 6, S. 708 (1885); Bd. 7, S. 474 (1886). 
dukte entstehen, die auch nach längerer Einwirkung der Süure noch nicht gespaltene Ciellobiosekomplexe enthalten.

Meine nüchste Aufgabe wird es sein, die Einwirkung der Schwefelsäure auf eine noch längere Zeit auszudehnen und die erhaltenen Präparate der Acetolyse zu unterwerfen.

Ich will noch bemerken, daß ich die Acetolyse von verschiedenen Polysacchariden versuchte, um irgend welche krystallisierte Produkte der partiellen Hydrolyse zu erhalten, jedoch ohne guten Erfolg. Ich unterwarf der partiellen Hydrolyse Xylanpräparate aus Buchen und aus Robinienholz, das Mannan der Samen von Phytelephas macrocarpa (Steinnuß) und das Chitin. Obschon ich die Versuchsbedingungen variierte, ergaben die Acetolysen unbefriedigende amorphe Produkte.

\section{Experimenteller Teil.}

Darstellung des Ausgangsmaterials.

In ein Gemisch aus $1800 \mathrm{~g}$ konzentrierter Schwefelsäure und $600 \mathrm{~g}$ Wasser, das auf $20^{\circ}$ abgekühlt war, trug ich unter Vermeidung jeder Temperaturerhöhung durch zweckmäßige Kühlung in kleinen Portionen unter ständigem Rühren $250 \mathrm{~g}$ reinste Watte ein. Die Operation bis zur vollständigen Lösung der Watte dauerte 1 Stunde und 40 Minuten. Um das Amyloid nach dem Verfahren von Flechsig ${ }^{1}$ ) zu erhalten, ließ ich das Reaktionsgemisch noch $5 / 4$ Stunden bei $20^{\circ}$ stehen, dann filtrierte ich $500 \mathrm{ccm}$ der. Flüssigkeit durch Glaswolle in dünnem Strahle in $4 \mathrm{l}$ Wasser, unter lebhaftem Umrühren. Der flockige Niederschlag wurde mit immer stärkerem Alkohol durch Dekantation gewaschen, abgesaugt, dann in heißem Alkohol suspendiert, $1 / 2$ Stunde am Rückflußkühler gekocht, abgesaugt, das Auskochen wiederholt und das Produkt nach vollständigem Auswaschen mit Alkohol unter vermindertem Druck über Phosphorpentoxyd getrocknet. Ausbeute $54 \mathrm{~g}$.

Um die tieferen Abbauprodukte zu gewinnen, ließ ich das Reaktionsgemisch noch 1 Stunde bei $20^{\circ}$ stehen, und da die

1) E. Flechsig, Über Darstellung und chemische Natur des Cellulosezuckers, Diese Zeitschrift, Bd. 7, S. 523 (1883). 
Flüssigkeit beim Eingießen in Wasser sich teilweeise löste, rührte ich $500 \mathrm{ccm}$ derselben in 31 96\% igen Alkohol ein, dann wurde das Produkt wieder so behandelt, wie es bei der Darstellung des Amyloids erfolgte. Das über Phosphorpentoxyd unter vermindertem Druck getrocknete Präparat ließ sich leicht zerreiben und bildete ein farbloses Pulver. Ausbeute $71 \mathrm{~g}$.

Nach weiterem vierstündigen Stehen bei $20^{\circ}$ wurde eine dritte Portion von $500 \mathrm{ccm}$ des Reaktionsgemisches in $3 \mathrm{l}$ Alkohol eingetragen und $54 \mathrm{~g}$. eines ebenfalls farblosen Pulvers gewonnen.

Der Kürze halber bezeichne ich die drei erhaltenen Präparate, die verschiedene. Stufen der partiellen Hydrolyse darstellen mit I, II und III. Die Präparate I und II lösten sich nur teilweise in Wasser.

\section{Die Acetolysen.}

Die Acetolysen wurden nach zwei verschiedenen Verfahren ausgeführt. Zunächst acetylierte ich nach einer Modifikation der ursprünglichen Methode von Skraup und König, später führte ich Versuche nach dem Verfahren von Klein ${ }^{1}$ ) aus. Letztere Methode gab viel eindeutigere Resultate, sodaß der Vorzug der Kleinschen Methode gegen die ältere sehr in Vordergrund tritt.

Die Acetolysen nach Skraup und König wurden wie folgt ausgeführt:

Das Ausgangsmaterial (12 g) wurde mit einem Gemisch aus $48 \mathrm{ccm}$. Essigsäureanhydrid, und $6,4 \mathrm{ccm}$ konzentrierter Schwefelsäure abgegossen. Das Acetylierungsgemisch wurde ohne Temperaturerhöhang bereitet: Jetzt wird die Reaktionsmasse geschüttelt und die Temperatur derselben unter vorsichtigem Erwärmen auf dem Wasserbade so geregelt, daß die Temperatur von $105^{\circ}$ möglichst bald erreicht wird. Die gelbbraune Flüssigkeit wird auf etwa $60^{\circ}$ abgekühlt und in dünnem Strahle in 11 kaltes Wasser gegossen. Der ausge-

1) Friedrich Klein, Beiträge zur Kenntnis des acetolytischen Abbaus der Cellulose, Zeitschrift f. angewandte Chemie, Bd. 25, S. 1409 (1912). 
schierlene Niederschlag wird wiederholt durch Dekantation mit Wasser gewaschen, wobei er krystallinisch wird. Das abgesaugte Produkt wird zwischen gehürtetem Filtrierpapier einem Druck von 50 Atmosphären ausgesetzt, dann zweimal aus heißem Alkohol umkrystallisiert. Die erhaltenen Präparate wurden analysiert, ihr Schmelzpunkt und Drehungsvermögen bestimmt, verseift und das Osazon des gebildeten Zuckers dargestellt.

Bei der Verseifung und Osazondarstellung verfuhr ich wie folgt. ${ }^{1}$ )

$0,5 \mathrm{~g}$ des Acetylproduktes wurden in $40 \mathrm{ccm}$ heißem Alkohol suspendiert, rasch abgekühlt, sofort $1,5 \mathrm{ccm} 33 \%$ iger Kalilauge zugesetzt, 5 Minuten geschüttelt, und langsam, in kleinen Portionen Wasser zugesetzt, bis völlige Lösung eintrat. Man erhält ungefähr $45 \mathrm{ccm}$ Flüssigkeit, die zur Vervollständigung der Verseifung 24 Stunden beiZimmertemperatur stehen gelassen werden. Man neutralisiert jetzt mit Essigsäure und verdampft die Lösung unter vermindertem Druck auf etwa $6 \mathrm{ccm}$. Nach Zusatz von $0,5 \mathrm{~g}$ Phenylhydrazinchlorhydrat wird $1^{1 / 2}$ Stunden im Wasserbade erhitzt. Beim Erwärmen schieden sich in keinem Falle Krystallmassen aus, sondern die Flüssigkeit nahm eine tiefgelbe Färbung an. Dies beweist, daß d-Glukose in nachweisbaren Mengen nicht vorhanden war. Beim Erkalten begann die Ausscheidung von Phenylcellobiosazon. Nach dem Ümkrystallisieren aus heißem Wasser wurde der Schmelzpunkt des Präparates genommen.

Um mich über die Zusammensetzung der Mutterlaugen des Cellobioseoktacetats zu orientieren, bestimmte ich das Drehungsvermögen der auf $250 \mathrm{ccm}$ mit Alkohol verdünnten Proben. Das spezifische Gewicht der Lösung wurde aus dem Gewichte ron $100 \mathrm{ccm}$ der Flüssigkeit, und der Prozentgehalt durch Eindampfen derselben in einer Platinschale auf dem Wasserbade ermittelt.

Hier sollen die Ergebnisse der Acetolysen folgen:

1) Emil Abderhalden und Géza Zemplén, Partielle Hydrolỵse der Tunicatencellulose. Bildung von Cellobiose. Diese Zeitschrift, Bd. 72 S. 58-62 (1911). 


\section{Präparat 1.}

Ausbeute nach dem Auspressen unter 50 Atmosphären Drück: $20 \mathrm{~g}$. Das erste Umkrystallisieren geschah aus $120 \mathrm{ccm}$, das zweite aus $200 \mathrm{ccm} 96 \%$ igem Alkohol. Erhalten $1,9 \mathrm{~g}$ Substanz. Aus farblosen Nadeln bestehende homogene Masse. Das Präparat sintert bei $225^{\circ}$ und schmilzt bei $228^{\circ}$ zu einer farblosen Flüssigkeit.

$0,2062 \mathrm{~g}$ gaben $0,3769 \mathrm{~g} \mathrm{CO}_{2}$ und $0,1088 \mathrm{~g} \mathrm{H}_{2} \mathrm{O}$.

Berechnet für Oktacetylcellobiose $\mathrm{C}_{28} \mathrm{H}_{38} \mathrm{O}_{19}(676,29)$ :

$$
49,54 \% \mathrm{C} ; 5,65 \% \mathrm{H}
$$

Gefunden: $\quad 49,85 \% \mathrm{C} ; 5,90 \% \mathrm{H}$.

$0,2945 \mathrm{~g}$ in Chloroform gelöst; Gesamtgewicht der Lösung $12,4373 \mathrm{~g}$ spezifisches Gewicht bei $20^{\circ}: 1,472 ;$ drehte Natriumlicht im $1 \mathrm{dm}-$ Rohr um $+1,37^{\circ}$ nach rechts; mithin:

$$
[\alpha]_{D}^{20}=+39,4^{0} \text { in Chloroform. }
$$

Das Präparat gab 0,06 g Phenylcellobiosazon, das beim raschen Erhitzen gegen $195^{\circ}$ unter Braunfärbung und Gasentwicklung schmolz.

Drehungsvermögen der ersten Mutterlauge:

4,138 g Substanz in Alkohol gelöst; Gesamtgewicht der Lösung $83,19 \mathrm{~g}$; spezifisches Gewicht $0,8338 \mathrm{~g}$; drehte Natriumlicht in $1 \mathrm{dm}-\mathrm{Rohr}+1,55^{\circ}$ nach rechts; mithin

$$
[\alpha]_{D}^{20}=+37,4^{\circ} \text { in Alkohol. }
$$

\section{Präparat II.}

Das Rohprodukt nach dem Auspressen unter 50 Atmosphären Druck betrug $20 \mathrm{~g}$. Das erste Mal wurde aus $70 \mathrm{ccm}$, das zweite Mal aus $120 \mathrm{ccm}$ Alkohol umkrystallisiert. Erhalten 0,94 g einer krystallinischen Substanz, die unter dem Mikroskop die deutliche F'orm der einzelnen Krystalle nicht erkennen ließ. Das Produkt sinterte gegen $200^{\circ}$ und schmolz gegen $215^{\circ} \mathrm{zu}$ einer dunkelgelben Flüssigkeit.

$0,2354 \mathrm{~g}$ gaben $0,4334 \mathrm{~g} \mathrm{CO}_{2}$ und $0,1242 \mathrm{~g} \mathrm{H}_{2} \mathrm{O}$.

Berechnet für Oktacetylcellobiose $\mathrm{C}_{28} \mathrm{H}_{38} \mathrm{O}_{19}(676,29 \mathrm{~g})$ :

$$
49,54 \% \mathrm{C} ; 5,65 \% \mathrm{H}
$$

Gefunden: $\quad 50,21 \% \mathrm{C} ; 5,90 \% \mathrm{H}$. 
$0,3310 \mathrm{~g}$ in Cihloroform gelöst; Gesamtgewicht der Lösung $12,2847 \mathrm{~g}$; spezifisches Gewicht 1,471 ; drehte bei $20^{\circ}$ Natriumlicht im 1 dun-Rohr um $+1,06^{\circ}$ rach rechts, mithin

$$
[a]_{0}^{20)}=+-26,4^{0} \text { in Chloroform. }
$$

Dus Produkt lieferte $0,05 \mathrm{~g}$ unreines Phenylcellobiosazon, das beim raschen Erhitzen gegen $180^{\circ}$ unter Braunfärbung und Gasentwicklung schmol\%.

Drehungsvermögen der ersten Mutterlauge:

2,388 $\mathrm{g}$ in Alkohol gelöst; Gesantgewicht der Lösung $82,16 \mathrm{~g}$, spezifisches Gewicht 0,8231 ; drehte bei $20^{\circ}$ Natriumlicht im $1 \mathrm{dm}$-Rohr um $0,74^{\circ}$ nach rechts: mithin

$$
\begin{gathered}
{[\alpha]_{\mathrm{D}}^{20}=+30,9^{\circ} \text { in Alkohol. }} \\
\text { Präparat III. }
\end{gathered}
$$

Rohprodukt nach dem Auspressen unter 50 Atmosphären Druck: 20 g. Das erste Umkrystallisieren geschah aus $70 \mathrm{ccm}$, das zweite aus $120 \mathrm{ccm}$ Alkohol. Erhalten 0,70 g an krystallisierter Substanz. Die verschiedene Größe der langen Prismen läßt erkennen, daß die Masse nicht vollkommen einheitlich ist. Das Produkt sintert bei $215^{\circ}$ und schmilzt vollständig bei $221^{\circ}$ zu einer hellgelben Flüssigkeit.

$0,1966 \mathrm{~g}$ gaben $0,3597 \mathrm{~g} \mathrm{CO}_{2}$ und $0,1029 \mathrm{~g} \mathrm{H}_{2} \mathrm{O}$.

Berechnet für Oktacetylcellobiose $\mathrm{C}_{28} \mathrm{H}_{38} \mathrm{O}_{19}(676,29)$ :

$$
49,54 \% \mathrm{C} ; 5,65 \% \mathrm{H}
$$

Gefunden: $\quad 49,90 \% \mathrm{C} ; 5,86 \% \mathrm{H}$.

$0,3614 \mathrm{~g}$ in Chloroform gelöst; Gesamtgewicht $11,9806 \mathrm{~g}$, spezifisches Gewicht 1,470 ; drehte Natriumlicht bei $20^{\circ}$ in $1 \mathrm{dm}$-Rohr um $+1,41^{\circ}$ nach rechts; mithin

$$
[\alpha]_{\mathrm{D}}^{20}=+31,8^{\circ} \text { in Chloroform. }
$$

Das Produkt lieferte 0,05 g Cellobiosazon, das beim raschen Erhitzen gegen $190^{\circ}$ unter Braunfärbung und Gasentwíkcklung schmolz.

Drehungsvermögen der ersten Mutterlauge:

3,008 $\mathrm{g}$ in Alkohol gelöst; Gesamtgewicht der Lösung $83,06 \mathrm{~g}$, spezifisches Gewicht $0,8321 \mathrm{~g}$; drehte Natriumlicht bei $20^{\circ}$ im $1 \mathrm{dm}-$ Rohr um $+0,81^{\circ}$ nach rechts; mithin

$$
[\alpha]_{\mathrm{D}}^{20}=+26,9^{\circ} \text { in Alkohol. }
$$


Das Ergebnis der Versuche ist nicht ganz befriedigend. Zwar gaben alle drei acetylierte Präparate bei der Verseifung Cellobiose bezw. Phenylcellobiosazon, die Menge und der Reinheitsgrad des letzteren waren aber sehr verschieden. Die reinste Oktacetylverbindung wurde aus Präparat I erhalten, dann folgte Präparat III, endlich Präparat II.

Dieselbe Reihenfolge tritt klar hervor bei der Betrachtung der Schmelzpunkte, und der Ergebnisse der Analysen und der Bestimmungen des Drehungsvermögens. Die Versuchsreihe zeigt, daß in allen drei Fällen Cellobiose gebildet wurde.

Dasselbe Ergebnis wird verstärkt und mit aller Strenge bewiesen durch die Acetolysen nach Klein, wobei die Mengenverhältnisse der gebildeten Cellobiose ebenfalls vergleichbar werden.

$10 \mathrm{~g}$ des Ausgangsmaterials werden mit $50 \mathrm{~g}$ eines Acetylierungsgemisches übergossen, das $80 \%$ Essigsäureanhydrid und 20\% konzentrierte Schwefelsäure enthält. Die Bereitung der Acetylierungsflüssigkeit geschah ohne merkliche Temperaturerhöhung. Das Reaktionsprodukt wird unter fortwährendem Rühren und Kühlung unter $30^{\circ}$ gehalten, bis die Masse zu einem homogenen Öl umgewandelt wird. Jetzt werden die Proben mit eingeschliffenem Glasstöpsel versehen 10 Tage bei Zimmertemperatur aufbewahrt. Von Zeit zu Zeit wird das Reaktionsgemisch mit einem Glasstabe tüchtig umgerührt. In jeder Probe erschien nach etwa 7 Tagen eine krystallisierte Ausscheidung, die der Reaktionsmasse eine dünne, breiartige Konsistenz verlieh. Der Inhalt der Gefäße wurde mit je $20 \mathrm{ccm}$ Eisessig verrührt und in 11 Wasser gegossen, wobei ein nahezu farbloser, flockiger Niederschlag . ausfiel. Durch Dekantation wurde derselbe ausgewaschen, dann abgesaugt, gepreßt und zweimal aus heißem Alkohol umkrystallisiert.

\section{Präparat I.}

Die Ausbeute nach dem Auspressen unter 50 Atmosphären Druck betrug $10 \mathrm{~g}$. Bei der ersten Krystallisation wurden $200 \mathrm{ccm}$, bei der zweiten $280 \mathrm{ccm}$ Alkohol verbraucht. 
Erhalten 2,01 g einer sehr einheitlichen, aus langen, mit freiem Auge sichtbaren, farblosen Nadeln bestehenden Substanz. Das Produkt sintert bei $220^{\circ}$ und schmilzt vollständig bei $224^{\circ}$ zu einer farblosen Flüssigkeit.

$0,2102 \mathrm{~g}$ gaben $0,3838 \mathrm{~g} \mathrm{CO}_{2}$ und $0,1094 \mathrm{~g} \mathrm{H}_{2} \mathrm{O}$.

Berechnet für Oktacetylcellobiose $\mathrm{C}_{28} \mathrm{H}_{38} \mathrm{O}_{19}(676,29)$ :

$49,54 \% \mathrm{C} ; 5,65 \% \mathrm{H}$.

Gefunden: $\quad 49,80 \% \mathrm{C} ; 5,82 \% \mathrm{H}$.

$0,4189 \mathrm{~g}$ Substanz in Chloroform gelöst; Gesamtgewicht der Lösung $13,4011 \mathrm{~g}$; spezifisches Gewicht 1,470; drehte Natriumlicht bei $20^{\circ}$ in $1 \mathrm{dm}$-Rolır um $+1,92^{\circ}$ nach rechts; mithin:

$$
[a]_{D}^{20}=+41,8^{\circ} \text { in Chloroform. }
$$

Das Produkt liefert $0,07 \mathrm{~g}$ Phenylcellobiosazon, das bei raschem Erwärmen gegen $198^{\circ}$ unter Braunfärbung und Gasentwicklung sich zersetzt.

Drehungsvermögen der ersten Mutterlauge:

$1,468 \mathrm{~g}$ Substanz in Alkohol gelöst; Gesamtgewicht der Lösung $81,52 \mathrm{~g}$; spezifisches Gewicht 0,8166 ; drehte Natriumlicht bei $20^{\circ}$ in $1 \mathrm{dm}-$ Rohr um $+0,59^{\circ}$ nach rechts; mithin:

$$
[\alpha]_{D}^{20}=+40,30^{\circ} \text { in Alkohol. }
$$

\section{Präparat II.}

Rohausbeute nach dem Pressen unter 50 Atmosphären Druck: 8 g. Das erste Umkrystallisieren geschah aus $150 \mathrm{ccm}$, das zweite aus $250 \mathrm{ccm}$ Alkohol. Gewonnen 1,41 g einer absolut einheitlichen, aus langen, seidenglänzenden, farblosen Nadeln bestehenden Substanz. Das Präparat sintert bei $220^{\circ}$ und schmilzt bei $225^{\circ} \mathrm{zu}$ einer farblosen Flüssigkeit.

$0,2365 \mathrm{~g}$ Substanz gaben $0,4322 \mathrm{~g} \mathrm{CO}_{2}$ und 0,1266 $\mathrm{g} \mathrm{H}_{2} \mathrm{O}$.

Berechnet für Oktacetylcellobiose $\mathrm{C}_{28} \mathrm{H}_{38} \mathrm{O}_{19}(676,29)$ :

$$
49,54 \% \mathrm{C} ; 5,65 \% \mathrm{H} \text {. }
$$

Gefunden: $\quad 49,84 \% \mathrm{C} ; 5,99 \% \mathrm{H}$.

0,4979 g Substanz in Chloroform gelöst; Gesamtgewicht 'der Lösung 13,1572 g; spezifisches Gewicht 1,470; drehte Natriumlicht bei $20^{\circ} \mathrm{im} 1 \mathrm{dm}-$ Rohr um $+2,32^{\circ}$ nach rechts; mithin:

$$
[\alpha]_{D}^{20}=+41,7^{\circ} \text { in Chloroform. }
$$


Das Produkt liefert $0,07 \mathrm{~g}$ Phenylcellobiosazon, das beim raschen Erwärmen gegen $196^{\circ}$ unter Zersetzung und Gasentwicklung schmilzt.

Drehungsvermögen der ersten Mutterlauge:

$1,168 \mathrm{~g}$ Substanz in Alkohol gelöst; Gesamtgewicht der Lösung $81,28 \mathrm{~g}$; spezifisches Gewicht 0,8142 ; drehte Natriumlicht bei $20^{\circ} \mathrm{im} 1 \mathrm{dm}-$ Rohr um $+0,49^{\circ}$ nach rechts; mithin :

$$
[\alpha]_{\mathrm{D}}^{20}=+41,9^{\circ} \text { in Alkohol. }
$$

\section{Präparat III.}

Rohausbeute nach dem Pressen unter 50 Atmosphären Druck: $10 \mathrm{~g}$. Bei der ersten Krystallisation wurden $170 \mathrm{ccm}$, bei der zweiten $260 \mathrm{ccm}$ Alkohol verbraucht. Gewonnen 1,92 $\mathrm{g}$ einer Substanz, die in Krystallform und Schmelzpunkt völlig mit dem aus Präparat II erhaltenen Acetylderivat übereinstimmte.

$0,2466 \mathrm{~g}$ gaben $0,4508 \mathrm{~g} \mathrm{CO}_{2}$ und $0,1274 \mathrm{~g} \mathrm{H}_{2} \mathrm{O}$.

Berechnet für Oktacetylcellobiose $\mathrm{C}_{28} \mathrm{H}_{38} \mathrm{O}_{19}(676,29)$ :

$$
49,54 \% \mathrm{C} ; 5,65 \% \mathrm{H} \text {. }
$$

Gefunden: $\quad 49,86 \% \mathrm{C} ; 5,78 \% \mathrm{H}$.

0,3982 g Substanz in Chloroform gelöst; Gesamtgewicht der Lösung 13,1722 g; spezifisches Gewicht 1,470; drehte Natriumlicht bei $20^{\circ} \mathrm{im} 1 \mathrm{dm}-\mathrm{Rohr}$ um $+1,90^{\circ}$ nach rechts; mithin:

$$
[\alpha]_{\mathrm{D}}^{20}=+42,7^{\circ} \text { in Chloroform. }
$$

Das Produkt lieferte 0,065 g Phenylcellobiosazon, das beim kurzen Erwärmen gegen $198^{\circ}$ unter Zersetzung und Gasentwicklung schmolz.

Drehungsvermögen der ersten Mutterlauge:

$1,818 \mathrm{~g}$ Substanz in Alkohol gelöst; Gesamtgewicht der Lösung 81,40 ; spezifisches Gewicht 0,8155 ; drehte Natriumlicht bei $20^{\circ}$ im $1 \mathrm{dm}-R o h r$ um $+0,77^{\circ}$ nach rechts; mithin:

$$
[\alpha]_{\mathrm{D}}^{20}=+42,3^{\circ} \text { in Alkohol. }
$$

Die charakteristischen Konstanten der Oktacetylcellobiose, sowie des Phenylcellobiosazons zeigen bei der zweiten Versuchsreihe sowohl untereinander, als mit den in der Literatur befindlichen zuverlässigen Werten eine sehr gute Übereinstim- 
mung. Demnach beweist diese zweite Versuchsreihe, daß aus den drei untersuchten Präparaten bei der Acetolyse Oktacetylcellobiose entstanden ist, und zwar in Mengen, die sich der Hälfte derjenigen aus normalen Cellulosen oder aus Hỵdrocellulosen erreichbaren Oktacetylcellobioseausbeuten nähern. Während Watte und Hydrocellulosen bei der Acetolyse nach Klein 50-55\% Oktacetylcellobiose liefern, entstand aus meinen Präparaten 15-20\% der Acetylverbindung auf das Ausgangsmaterial berechnet. Es ist sehr auffallend, daß das Amyloid und das dritte Präparat, das bei längerer Einwirkung der Schwefelsäure (6 Stunden) entstand, nahezu dieselben Cellobioseausbeuten lieferten. Der Befund spricht dafür, daß die Einwirkung der Schwefelsäure unter den angegebenen Konzentrationsbedingungen sich nicht auf die Hydrolyse der Cellobiose erstreckt. Diese Vermutung wird durch das optische Verhalten der ersten Mutterlaugen nach der Ausscheidung der Oktacetylcellobiose aus der. heißen, alkoholischen Flüssigkeit verstärkt. Das Drehungsvermögen der Mutterlaugen ist nämlich nie hoch genug, um auf die Anwesenheit größerer Mengen von $\alpha$-Pentacetylglukose schließen zu können. Wenn bei der längeren Einwirkung der starken Schwefelsäure d-Glukose gebildet wäre, so müßte diese unter den Bedingungen der Acetolyse in $\alpha$-Pentacetylglukose umgewandelt werden. Um die Grundlage dieses Gedankenganges $\mathrm{zu}$ verstärken, habe ich besondere Versuche mit d-Glukose ausgeführt, um zu erfahren, welche der beiden Isomeren der Pentacetylglukose dabei entsteht.

$5 \mathrm{~g} \mathrm{~d}$-Glukose werden mit $20 \mathrm{ccm}$ eines Acetylierungsgemisches übergossen, das aus $150 \mathrm{ccm}$ Essigsäureanhydrid und $5 \mathrm{ccm}$ konzentrierter Schwefelsäure unter Kühlung bereitet war. Die Mengen der in Reaktion tretenden Substanzen sind so gewählt, daß beim Eintreten der Reaktion die Erwärmung der Masse auf $100^{\circ}$, aber nicht höher, erfolgt. Der in Lösung gegangene Zucker wird in $150 \mathrm{ccm}$ Wasser gegossen. Das ausgeschiedene hellgelbe Öl erstarrt rasch.zu einer harten, krystallinischen Masse, die abgesaugt, mit Wasser gewaschen, gepreßt und aus $40 \mathrm{ccm}$ heißem Alkohol umgelöst wird. Ausbeute $4,2 \mathrm{~g}$; Schmelzpunkt $112^{\circ} ;[\alpha]_{\mathrm{D}}^{20}=+102^{\circ}$ in Chloroform. 
Diese Konstanten sind für die $\alpha$-Pentacetylglukose charakteristisch. Wenn diese Verbindung in erheblichen Mengen bęi der Acetolyse der untersuchten Produkte entstanden wäre, so müßte dies in dem Drehungsvermögen der ersten Mutterlaugen der Oktacetylcellobiose Ausdruck finden. Das Drehungsvermögen der alkoholischen Mutterlaugen ist aber ungefähr dasselbe als dasjenige der reinen Oktacetylcellobiosen. Man muß aber nicht vergessen, daß das Drehungsvermögen der Oktacetylcellobiosen in Chloroformlösung ermittelt wurde. Eine . Bestimmung des Drehungsvermögens in alkoholischer Lösung war aber wegen der Schwerlöslichkeit des Produktes in diesem Lösungsmittel nicht ausführbar. Deshalb blieb nichts anderes übrig, als das Drehungsvermögen der in Alkohol bezw. Chloroform gelösten Substanzen zu vergleichen. Dadurch habe ich keinen so groben Fehler begangen, daß die daraus gefolgerten Schlüsse illusorisch werden. 